\title{
MOLECULAR MASS DISTRIBUTION IN THE OUTER
}

\section{GALAXY}

\author{
SEAN J. CAREY \\ Rensselaer Polytechnic Institute, Troy, NY, 12180, USA \\ carey@charon.phys.rpi.edu
}

AND

KATHRYN N. MEAD AND MARC L. KUTNER

NRAO, Tucson, AZ, USA

\section{Introduction}

The extent and nature of molecular material in the outer Galaxy $\left(R>R_{\odot}\right)$ has been an open, interesting question since the discovery of molecular material in the outer Galaxy (Kutner \& Mead, 1981). From large scale CO surveys, two estimates of the total molecular mass have been made with the same result, $\mathrm{M}\left(\mathrm{H}_{2}\right)=6 \times 10^{8} \mathrm{M}_{\odot}$ (Wouterloot et al., 1990), (Digel et al., 1990). Both estimates have a strong bias towards large, star forming (warm) clouds. Wouterloot et al. used the IRAS point source catalog as a finder chart for their observations and the inner Galaxy mass distribution to scale their result. Digel et al. conducted an unbiased survey with a large (8.8 arcminute) beam which underestimated the amount of material in small clouds due to the effects of beam dilution.

Recent observations by Lequeux et al. (1993) have spurred interest in the amount of molecular material contained in small, cold molecular clouds. Their results based on $\mathrm{CO}$ absorption measurements along 2 lines of sight indicate the presence of cold molecular gas. However, their suggestion that cold molecular gas is 4-5 times more abundant than HI in the outer Galaxy is based on the tenuous assumption that the ratio of molecular to atomic column densities at a particular velocity is indicative of the ratio for all $\mathrm{R}>\mathrm{R}_{\odot}$ (Peters \& Bash, 1987).

We have recently completed the most sensitive survey to date in ${ }^{12} \mathrm{CO}(\mathrm{J}=1 \rightarrow 0)$ for $\mathrm{R}>\mathrm{R}_{\odot}$ using the NRAO $12 \mathrm{~m}$ (Carey et al., 1994). Two cloud populations, arm (at $\mathrm{R} \sim 13 \mathrm{kpc}$ ) and interarm, have been identified. The data provide us with an unbiased probe of the small and/or cold 
molecular cloud component. For the range of galactocentric radii covered, $9 \mathrm{kpc}<\mathrm{R}<16 \mathrm{kpc}$, our survey is complete for clouds with $\mathrm{T}_{\mathrm{K}} \geq 5 \mathrm{~K}$ and $\mathrm{r} \geq 2 \mathrm{pc}$.

\section{Correction to Total Mass Determination}

Our observations have identified a small ( $\mathrm{r}<5 \mathrm{pc}$ ) molecular cloud component in the region surveyed. These clouds appear to be significantly $\mathrm{CO}$ underluminous when compared to their large, inner Galaxy counterparts. This result may be partially due to beam dilution, but it also indicates that the conversion factor (X) between CO luminosity and cloud mass is higher in the outer Galaxy (Mead \& Kutner, 1988). As the X factor for these clouds is uncertain and the clouds resemble other molecular clouds which are most likely virialized, we use the virial theorem to calculate cloud masses. The breakdown of the observed mass distribution as a function of cloud size and environment is given below.

Total Mass Distribution in units of $10^{5} M_{\odot}$

\begin{tabular}{lccc}
\hline & \multicolumn{3}{c}{ Cloud Size } \\
& $\mathrm{r}<5 \mathrm{pc}$ & $\mathrm{r} \geq 5 \mathrm{pc}$ & Total \\
\hline Arm Population & 1.8 & 6.8 & 8.6 \\
Interarm Population & 1.4 & 0.7 & 2.1 \\
Total & 3.2 & 7.5 & 10.7 \\
\hline
\end{tabular}

Fully $30 \%$ of the observed mass is contained in small (cold) clouds. If the survey region is a representative sample of the outer Galaxy, then previous estimates could possibly be scaled upward by $30 \%$; however, there is little evidence to support the suggestion of Lequeux et al. that cold molecular material is a gravitationally significant component of the outer Galaxy mass distribution. If small, cold clouds exist out to $\mathrm{R} \sim 25 \mathrm{kpc}$ then the total molecular mass could increase by a factor of 2 .

S. Carey received support from the Astronomical Society of New York and Sigma Xi, The Scientific Research Society.

\section{References}

Carey, S. J., Mead K. N., \& Kutner, M. L. 1994, ApJ, in preparation

Digel, S., Bally, J., \& Thaddeus, P. 1990, ApJ, 357, 29

Peters, W. L., \& Bash, F. N. 1987, ApJ, 317, 646

Lequeux, J., Allen, R. J., \& Guilloteau, S. 1993, A\&A, 280, L23

Kutner, M. L. \& Mead, K. N. 1981, ApJ, 249, L15

Mead, K. N., \& Kutner, M. L. 1988, ApJ, 330, 399

Wouterloot, J. G. A., Brand, J., Burton, W. B., \& Kwee K. K. 1990, A\&A, 230, 21 